\title{
Serotypes and Antibiotic Resistance of Streptococcus pneumoniae from Adenoids in Preschool Children with Recurrent Upper Respiratory Tract Infections
}

\author{
IZABELA KORONA-GLOWNIAK ${ }^{1 *}$, ARTUR NIEDZIELSKI ${ }^{2}$, ANNA MALM $^{1}$ and GRAŻYNA NIEDZIELSKA ${ }^{3}$ \\ ${ }^{1}$ Department of Pharmaceutical Microbiology, Medical University of Lublin, Lublin, Poland \\ ${ }^{2}$ Otoneurology Lab III Chair of Paediatric, Medical University of Lublin, Lublin, Poland \\ ${ }^{3}$ Department of Pediatric Otolaryngology, Phoniatrics and Audiology, Medical University of Lublin, Lublin, Poland
}

Submitted 28 March 2013, revised 29 April 2013, accepted 3 September 2013

Abstract

We investigated children aged 2-5, who had gone adenoidectomy for recurrent and/or persistent symptoms of upper respiratory tract infections for prevalence of pneumococci in adenoid tissue. Serotypes and antibiotic resistance patterns of the isolated pneumococci were determined and also risk factors of pneumococcal colonization were defined. S. pneumoniae colonization in adenoids was found in $62(60.2 \%)$ children. Serotypes belonged to 10 -valent and 13-valent pneumococcal conjugated vaccines (PCVs) constituted 56.1\% and $68.2 \%$ of the isolates, respectively. Decreased susceptibility to penicillin was found in $45.5 \%$ of isolates; pneumococci were resistant to cotrimoxazole (62.1\%), tetracycline (43.9\%), erythromycin (54.5\%), clindamycin (54.5\%) and chloramphenicol (31.8\%). Multidrug resistant S. pneumoniae comprised $57.6 \%$ of the isolates. Antibiotic resistant pneumococci were mostly distributed among serotypes belonged to 10 -valent and 13-valent PCVs. Good vaccine coverage among the isolated pneumococci confirmed that the introduction of PCVs in the national immunization programme may reduce the pool of resistant and multidrug resistant pneumococci in a community.

Ke y words: Streptococcus pneumoniae, adenoids, antibiotic resistance patterns, serotypes

\section{Introduction}

Upper respiratory tract infections (URTIs) are the most common childhood illnesses but remains clinically indistinguishable whether it is of viral or bacterial etiology. However, URTIs are crucial cause of visits in family doctor practice, and the major cause of antibiotic prescriptions, especially by pediatricians. Streptococcus pneumoniae is an important etiologic agent of meningitis, pneumonia, bacteriemia and acute otitis media in both children and adults and one of the major bacterial pathogens colonizing nasopharynx, mainly asymptomatically (Bogaert et al., 2004). Young children are colonized the most frequently and they have been found to be main reservoir of pneumococci, paying the key role in spreading and selecting of multidrug resistant strains (De Lencastre and Tomasz, 2002). Recently, there has been an increasing rate of antibiotic resistance in the pneumococcal serotypes that are responsible for the infections of middle ear, nasal cavity and pharynx in children and are causing difficulties in the treatment (Appelbaum, 2002). In fact, a fundamental pneumococcal infections are of the respiratory tract and not invasive diseases in spite of the common belief by many pediatricians.

Despite the advances in the development of pneumococcal conjugate vaccines (PCVs) leading to a reduction of invasive disorders, eradication of pneumococcal diseases is not within easy reach. The first introduced conjugate vaccine contains seven pneumococcal capsular antigens (PCV7) covering serotypes 4, 6B, 9V, 14, $18 \mathrm{C}, 19 \mathrm{~F}$ and $23 \mathrm{~F}$. Its impact on the decrease of invasive pneumococcal disease has been significant both in infants, older children and adults. Additional benefit of the vaccine was a decrease in rates of antimicrobial resistance among pneumococcal isolates resulting from association resistance to penicillin, macrolides and multidrug resistance with serotypes $6 \mathrm{~B}, 9 \mathrm{~V}, 14,19 \mathrm{~F}$ and 23F included to PCV7 (O'Brien et al., 2009). In 2007, World Health Organization (WHO) recommended all countries to incorporate PCV to their national infant immunization program. Only in countries with routine effective use of PCV7, the proportion of current coverage of serotypes belonged to PCV7 is lower than in the pre-vaccine era (Isaacman et al., 2010). PCV-7 was introduced in Poland in 2005 and recommended for

* Corresponding author: I. Korona-Glowniak, Department of Pharmaceutical Microbiology, Medical University of Lublin, Chodzki 1 Street, 20-093 Lublin, Poland; phone: +48 8174237 73; fax: +48 8174237 72; e-mail: iza.glowniak@umlub.pl 
children under 2 years old but not refunded by health authorities. Since 2009, the PCV was recommended for children under 5 years old and refunded for some risk groups. According to our previous study, the adenoids like the nasopharynx can be regarded as a reservoir of S. pneumoniae, including resistant and/or multiresistant strains. As a continuation of that study we investigated pneumococci isolated from adenoid tissue of children aged 2-5, who had gone adenoidectomy for recurrent and/or persistent symptoms of upper respiratory tract infections. Serotypes and antibiotic resistance patterns of the isolated pneumococci were determined and also risk factors of adenoid colonization by pneumococci were defined.

\section{Experimental}

\section{Material and Methods}

Patients. The study enrolled 103 children, aged between 2 and 5, undergoing adenoidectomy in Department of Pediatric Otolaryngology, Phoniatrics and Audiology, Medical University of Lublin during MayJune and November-December 2011. The indication for adenoidectomy was recurrent acute pharyngotonsilitis for at least 2 years with 5 or more acute attacks per year. Patients didn't received any antibiotic therapy for at least 20 days before the operation. From all children's parents, the informed content were obtained. The Ethical Committee of the Medical University of Lublin approved the study protocol (No. KE-0254/75/211).
Demographic data of studied children was shown in Table I. None of the children were immunized by a pneumococcal vaccine.

Laboratory procedures. After the surgery, the adenoid were placed in the sterile container and were transported to laboratory then the adenoid was swabbed with sterile alginate-tipped applicator. Swabs were inoculated on selective Mueller-Hinton agar with $5 \%$ sheep blood and $0.5 \mathrm{mg} / \mathrm{L}$ of gentamicin for selective cultivation of streptococci. The streaked agar plates were incubated aerobically at $35^{\circ} \mathrm{C}$ in $5 \% \mathrm{CO}_{2}$ enriched atmosphere for 24 to 48 hours. Pneumococci were identified by colony morphology, susceptibility to optochin $(5 \mu \mathrm{g})$, and bile solubility; identification was confirmed by a slide agglutination test Slidex PneumoKit (BioMerieux).

All isolates were serotyped by means of Quellung reaction using antisera provided by Statens Serum Institute (Copenhagen, Denmark). We applied antisera for determination of serotypes belonging to the 23-valent pneumococcal polysaccharide vaccine (PPV23), i.e. - 1, 2, 3, 4, 5, 6B, 7F, 8, 9N, 9V, 10A, 11A, 12F, 14, 15B, 17F, 18C, $19 \Lambda, 19 \mathrm{~F}, 20,22 \mathrm{~F}, 23 \mathrm{~F}, 33 \mathrm{~F})$ and also serotypes $6 \Lambda, 23 \Lambda$. The isolates negative to employed pooled sera but positive to omni serum were defined as Others, serotypes not present in PPV23. The isolates negative to slide agglutination test and negative to omni serum were defined as untypeable (rough - R); their belonging to pneumococci was confirmed by PCR analysis using primers for detecting the lytA gene encoding the autolysin enzyme specific to S. pneumoniae (Simoes et al., 2011).

Table I

Demographic data of children undergoing adenoidectomy for recurrent URTIs.

\begin{tabular}{|c|c|c|c|}
\hline & sk factor & $\begin{array}{l}\text { Total number } \\
\text { of children (\%) }\end{array}$ & $\begin{array}{l}\text { SP colonized children } \\
\text { (\% in group) }\end{array}$ \\
\hline \multirow[t]{4}{*}{ Age (yr) } & 2 & $11(10.7)$ & $6(54.5)$ \\
\hline & 3 & $24(23.3)$ & $11(45.8)$ \\
\hline & 4 & $51(49.5)$ & $33(64.7)$ \\
\hline & 5 & $17(16.5)$ & $12(70.6)$ \\
\hline \multirow[t]{2}{*}{ Sex } & female & $43(41.7)$ & $26(60.5)$ \\
\hline & male & $60(58.3)$ & $36(60.0)$ \\
\hline \multirow[t]{3}{*}{ Sibling possessing } & no & $17(16.5)$ & $11(64.7)$ \\
\hline & 1 & $46(44.6)$ & $28(60.9)$ \\
\hline & $>2$ & $40(38.8)$ & $23(57.5)$ \\
\hline Passive smoking & & $32(31.1)$ & $19(59.4)$ \\
\hline DCC attendance & & $83(80.6)$ & $49(59.0)$ \\
\hline \multirow[t]{2}{*}{ Place of residence } & village & $41(39.8)$ & $23(56.1)$ \\
\hline & city & $62(60.2)$ & $39(62.9)$ \\
\hline \multirow{3}{*}{$\begin{array}{l}\text { Antibiotics taken } \\
\text { for the last attack }\end{array}$} & amoxicillin/co-amoxiclav & $55(54.5)$ & $34(61.8)$ \\
\hline & cephalosporins & $24(23.8)$ & $16(66.7)$ \\
\hline & macrolides & $22(21.8)$ & $10(45.5)$ \\
\hline
\end{tabular}

DCC, day care center; SP, Streptococcus pneumoniae 
Susceptibility of the isolates to oxacillin, erythromycin (E), tetracycline (Te), chloramphenicol (C), clindamycin (Cc), norfloxacin (Nor), rifampicin (Ra), teicoplanin (Tec), linezolid (Lzd) and trimethoprimsulfamethoxazole (Sxt) was determined by the disk diffusion method of Bauer and Kirby. Results were interpreted according to the European Committee on Antimicrobial Susceptibility Testing recommendations (EUCAST, 2011). Isolates exhibiting a zone of $\geq 20 \mathrm{~mm}$ around a $1 \mu \mathrm{g}$ oxacillin disk were reported as penicillin susceptible S. pneumoniae (PSSP); isolates exhibiting a zone of $<20 \mathrm{~mm}$ were further tested by the E-test (AB Biodisk, Sweden), following the manufacturer's instruction, to determine minimal inhibitory concentration (MIC) for benzylpenicillin. Isolates with MIC $\leq 0.064 \mathrm{mg} / \mathrm{L}$ were considered as fully susceptible to benzylpenicillin; isolates with MIC $>0.064 \mathrm{mg} / \mathrm{L}$ were called penicillin non-susceptible S. pneumoniae (PNSSP). Multidrug-resistant isolates of S. pneumoniae (MDR-SP) were defined as having resistance to at least 3 different classes of antibiotics. S. pneumoniae ATCC 49619 was used as control strain in the antimicrobial susceptibility tests.

Statistical analysis. Data processing and analysis were performed using StatSoft, Inc. STATISTICA 10. The potential predictor variables were tested in separate univariate analyses (Chi-squared or the Fisher exact test, as appropriate) for their association with upper respiratory colonization by S. pneumoniae in general, and by PNSSP or MDR-SP. Significant univariate predictors $(p<0.1)$ were tested for inclusion in the multivariate models, and nonsignificant variables were removed sequentially until only those significant at $\mathrm{p}<0.1$ remained. Variables of particular interest based on previous studies, such as children age, having siblings, passive smoking and type of antibiotic used, were included even when were not statistically significant. Statistical significance was set at $p<0.05$.

\section{Results}

S. pneumoniae colonization was observed in 62 $(60.2 \%)$ children who had undergone adenoidectomy for recurrent and/or persistent symptoms of upper respiratory tract infections. A total of 66 isolates were recovered; 4 (3.9\%) children were colonized by two different in colony morphology isolates, which were identified by serotyping and antimicrobial resistance tests as different pneumococcal strains. Among the isolates, serotypes belonged to PPV23 (89.4\%) were identified, and 4 isolates $(6.1 \%$ ) were untypeable (rough - R) (Table II).

The most frequent was serotype 19F (24.2\%). Serotypes belonged to pneumococcal conjugated vaccines

Table II

Serotypes and antibiotic resistance patterns of Streptococcus pneumoniae isolated from children undergoing adenoidectomy for recurrent URTIs.

\begin{tabular}{|l|c|l|}
\hline Serotypes & $\begin{array}{c}\text { No. (\%) } \\
\text { of isolates }\end{array}$ & \multicolumn{1}{|c|}{ Antibiotic resistance pattern (no. of isolates) } \\
\hline 3 & $5(7.6)$ & S (5) \\
\hline 6A & $2(3.0)$ & ECcTeSxt (1), S (1) \\
\hline 6B & $9(13.6)$ & PECcCTeSxt (2), PECcCSxt (4), PECcTeSxt (1), S (2) \\
\hline 9V & $2(3.0)$ & PSxt (2) \\
\hline 10A & $1(1.5)$ & CTe (1) \\
\hline 11A & $3(4.5)$ & S (3) \\
\hline 14 & $2(3.0)$ & PECcTeSxt (1), PECcSxt (1) \\
\hline 15B & $2(3.0)$ & Sxt (1), S (1) \\
\hline 15(nonB) & $4(6.1)$ & Sxt (2), S (2) \\
\hline 18C & $1(1.5)$ & S (1) \\
\hline 19A & $1(1.5)$ & PECcTeSxt (1) \\
\hline 19F & $16(24.2)$ & PECcCTeSxt (4), PECcTeSxt (4), ECcCTeSxt (1), ECcTeSxt (2), ECcTe (1), CTeSxt (1), CSxt (1), S (2) \\
\hline 23A & $1(1.5)$ & ECcCTe (1) \\
\hline 23B & $1(1.5)$ & S (1) \\
\hline 23F & $7(10.6)$ & PECcCTeSxt (1), PECcCSxt (4), PECcTeSxt (1), Sxt (1) \\
\hline 33F & $2(3.0)$ & ECcTe (2) \\
\hline Others & $3(4.5)$ & PECcTeSxt (1), S (2) \\
\hline R & $4(6.1)$ & PECcTeSxt (3), PCSxt (1) \\
\hline
\end{tabular}

P, penicillin; E, erythromycin; Cc, clindamycin; Te, tetracycline; C, chloramphenicol; Sxt, co-trimoxazol; S, sensitive to all tested antibiotics; R, rough, untypeable strain; Others, serotypes not present in PPV23. 


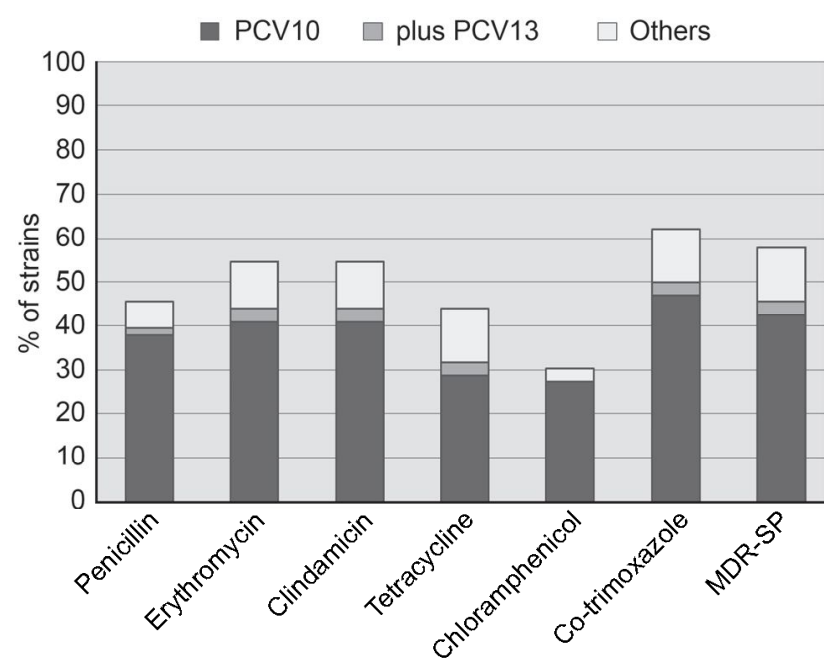

Fig. 1. Resistance rate to antibiotics of Streptococcus pneumoniae isolated from children undergoing adenoidectomy for recurrent URTIs.

Dark blocks, samples containing PCV10 type pneumococci; grey blocks, samples containing serotypes in PCV13 additional to PCV10 types; white blocks, samples containing non-vaccine type pneumococci.

- PCV10 (containing serotypes $1,4,5,6 \mathrm{~B}, 7 \mathrm{~F}, 9 \mathrm{~V}, 14$, 18C, 19F, 23F) and PCV13 (containing serotypes 3, $6 \mathrm{~A}, 19 \mathrm{~A}$ additionally to 10 -valent vaccine) constituted $56.1 \%$ and $68.2 \%$ of the isolates, respectively.

The pneumococcal isolates were susceptible to all tested antimicrobial agents in $30.3 \%$. These strains belonged to serotypes 3 ( 5 isolates), $11 \Lambda$ ( 3 isolates), $6 \mathrm{~B}, 19 \mathrm{~F}, 15$ non-B, NT ( 2 isolates per each serotype), and $6 \Lambda, 10 \Lambda, 15 \mathrm{~B}, 18 \mathrm{C}$ (1 isolate per each serotype). Among all of the strains, $45.5 \%$ had decreased susceptibility to penicillin (MIC range $0.12-2.0 \mathrm{mg} / \mathrm{L}, \mathrm{MIC}_{50}$ $0.5 \mathrm{mg} / \mathrm{L}$ and $\mathrm{MIC}_{90} 2.0 \mathrm{mg} / \mathrm{L}$ ). S. pneumoniae isolates were resistant to co-trimoxazole (62.1\%), tetracycline (43.9\%), erythromycin (54.5\%), clindamycin (54.5\%) and chloramphenicol (31.8\%) (Fig. 1). All isolates were susceptible to norfloxacin and according to EUCAST 2011, they should be reported as susceptible to levofloxacin and moxifloxacin and intermediate to ciprofloxacin and ofloxacin. None of the tested isolates was resistant to rifampicin, linezolid and teicoplanin. Each strain was characterized phenotypically by serotype and antibiotic resistance pattern (Table II). Multidrug resistance was present in $57.6 \%$ of isolates. Among MDRSP $76.3 \%$ were non-susceptible to penicillin. Antibiotic resistant pneumococci were mostly distributed among serotypes belonged to PCV10 and PCV13 (Fig. 1). PNSSP and MDR-SP strains represented PCV10 serotypes in $83.3 \%$ and $73.6 \%$, respectively and PCV13 serotypes in $86.7 \%$ and $78.9 \%$, respectively. Colonization with PNSSP and MDR-SP strains was found in $30(29.1 \%)$ and 35 (34.0\%) children, respectively.

During the analysis of risk factors predisposing to pneumococcal colonization, including PNSSP and
MDR strains, no predictors were found in the total population of children. However, when analysis in group of 32 children exposed to passive smoking was done, two predictors turned out to be significant for pneumococcal colonization: female gender $(\mathrm{p}=0.049$, OR $1.59,98 \% \mathrm{CI} 1.1-2.4)$ and type of antibiotics taken for the last attack $(\mathrm{p}=0.019)$. Moreover, in these children consumption of $\beta$-lactams increased the risk of pneumococcal colonization in comparison to macrolide consumption ( $\mathrm{p}=0.015$, RR 5.1, 95\%CI 1.2-20.8). Type of taken antibiotics was a predictor of MDR-SP colonization in children exposed to passive smoking $(\mathrm{p}=0.02)$. Macrolide consumption in these children decreased rate of MDR-SP colonization in comparison to children with consumption of $\beta$-lactams ( $\mathrm{p}=0.012$, RR 0.55, 95\%CI 0.4-0.8).

\section{Discussion}

The adenoids are involved in the pathology of adenotonsilitis, rhinosinusitis, otitis media and chronic nasal obstruction (Brook and Shah, 2001; Karlidag et al., 2002). High frequency of adenoid colonization by $S$. pneumoniae in preschool children with recurrent upper respiratory infections was found in our study, which is in agreement with the previous observations (Niedzielski et al., 2013). However, high rate of asymptomatic pneumococcal carriage in preschool children, especially attending day care center is common so it is difficult to indicate that $S$. pneumoniae is an important bacterial etiological agent of URTIs even though it is isolated from children with URTI symptoms (Gunnarsson et al., 2001). Jeong et al. (2007) analyzed the differences between the bacterial pathogens of tonsil core in recurrent tonsillitis and tonsillar hypertrophy with regard the age; S. pneumoniae was detected with high frequency in recurrent tonsillitis in the patients between $8-14$ years, even though this pathogen was more common in younger patients ( $<8$ years) in both groups. Syrjanen etal. (2001) reported that nasopharyngeal carriage of pneumococci during RTIs (without otitis media) in children increased from $13-43 \%$ to $45-56 \%$, depending on age. Contrary Greenberg et al. (2004) found no differences in the overall S. pneumoniae carriage between healthy and sick children in different age groups.

Resistant pneumococci are common among young children, especially attending day care centers. Transmission of these resistant strains in the community is constantly investigated (De Lencastre and Tomasz, 2002; Borer et al., 2001). Resistance to antimicrobial agents among pneumococcal isolates in this study was much higher than data concerning pneumococcal isolates described in Poland (Jacobs et al., 2003; Riedel 
et al., 2007). Children with recurrent pharyngotonsilitis are usually treated with multiple courses of antibiotics before surgery but many of them continue to carriage of pathogenic bacteria in the pharynx and the adenoids, including strains resistant to antibiotic (McCay, 2000) and all of children from our studies were treated by antibiotics before surgery. Poland belong to a part of Europe with high level of antibiotic consumption and above $20 \%$ rate of penicillin and macrolide resistance among the pneumococcal isolates (Riedel et al., 2007, van de Sande-Bruinsma et al., 2008).

The passive smoking increases colonization rate by pathogens in children and their risk for respiratory tract infections (Gryczynska et al., 1999; Kosikowska et al., 2011). Principi's et al. (1999) observation with healthy children indicated that exposure to tobacco smoke did not influence the $S$. pneumoniae carriage in the upper respiratory tract in children aged $<5$ years. Bakhshaee etal. (2012) found a significant difference in carriage rates between children who lives in smoking families compare to those with nonsmoking families for M. catarrhalis, but not for S. pneumoniae and H. influenzae. However, Greenberg et al. (2006) showed that exposure to tobacco smoke increased S. pneumoniae carriage rates particular in children. Data obtained in the present study indicated no influence of passive smoking on pneumococcal colonization in children with recurrent URTIs. However, contrary to our previous data (Niedzielski et al., 2013), in present paper no risk factor for pneumococcal colonization in total population of children undergoing adenoidectomy for recurrent URTIs was found, but in group of children exposed to passive smoking some predictors of the adenoid colonization by pneumococci were revealed - female gender as a predictor of pneumococcal colonization and type of antibiotics taken for last attack as a predictor of pneumococcal colonization and MDR-SP colonization. These observations suggest that the effect of passive smoking on pneumococcal prevalence in the upper respiratory tract, including adenoids, may be correlated with other predisposing factors, e.g. age, gender, season, staying in a close population (e.g. day care center), chronic respiratory infections, previous antibiotic treatment, allergy or geographic region.

Our data revealed that consumption of $\beta$-lactams increased the rate of pneumococcal colonization in adenoids but only in children exposed to tobacco smoke. On the other side the decrease of MDR-SP colonization was observed in this group of children who received macrolides. Some studies demonstrated an association between the use of a specific antibiotic and selective colonization with strains resistant to this drug as well as that number of courses of drugs to which pathogens are resistant has the utmost importance (Appel- baum, 2002). Borer et al. (2001) described statistically significant impact of antimicrobial drug use on nasopharyngeal carriage of S. pneumoniae, whereas Principi et al. (1999) showed macrolide therapy increased nasopharyngeal pneumococcal carriage. Findings from southern Israel strongly suggest that azithromycin affects increased multidrug resistance in $S$. pneumoniae (Barkai et al., 2005).

Widespread use of PCVs resulted in decreased in invasive pneumococcal disease and pneumonia among children and elderly persons in countries that have introduced conjugate vaccines (Center, 2008; Lopalco, 2009). In Poland PCV is already included to routine immunization program: vaccination is recommended universally for children $\leq 2$ years old and since 2009 is mandatory and refunded for some risk groups. However, low vaccination rate was observed, especially in south-east region of Poland. Our data showed that the S. pneumoniae serotype coverage of isolates colonizing adenoids in pre-school children with recurrent/ persistent URTIs from Poland by the currently available PCVs is high (56.1-68.2\%) and similar to that reported in other European countries (McIntosch et al., 2007). According to studies performed by Skoczynska et al. (2011), in children aged less than 5 years in Poland, serotypes $14,6 \mathrm{~B}$, and $19 \mathrm{~F}$ were most prevalent, comprising $52.7 \%$ of the invasive pneumococcal disease (IPD) cases. The PCV10, and PCV13 covered 54.8\%, and $68.8 \%$ of all IPD cases, and $76.3 \%$, and $86.3 \%$ of cases involving children under 5 years of age. An encouraging finding of our present data was also that a majority of PNSSP and MDR-SP belonged to serotypes included in PCV13 and PCV10. The above data suggest that routine vaccination of infants with PCVs could effectively reduce the reservoir of pneumococci within upper respiratory tract, including resistant and/ or multidrug resistant strains, in children in Poland, similarly to that in other European countries (O'Brien et al., 2009).

High carriage rate of S. pneumoniae in adenoids, including multidrug resistant strains, was observed in our study in children with an indication for adenoidectomy due to recurrent upper respiratory tract infections refractory to antibiotic therapy. Good vaccine coverage among the isolated pneumococci allows the suggestion that the introduction of PCVs in the national immunization program in young children may reduce the high carriage rate of pneumococci, colonizing not only nasopharynx but also adenoids.

\section{Acknowledgements}

The paper was developed using the equipment purchased within the agreement No. POPW.01.03.00-06-010/09-00 Operational Program Development of Eastern Poland 2007-2013, Priority Axis I, Modern Economy, Operations 1.3. Innovations Promotion. 


\section{Literature}

Appelbaum P.C. 2002. Resistance among Streptococcus pneumoniae: implications for drug selection. Clin. Infect. Dis. 34: 1613-20. Bakhshaee M., H.R. Naderi, K. Ghazvini, K. Sotoudeh, A. Amali and S.J. Ashtiani. 2012. Passive smoking and nasopharyngeal colonization by Streptococcus pnetmoniae, Haemophilus influenzae, and Moraxella catarrhalis in daycare children. Eur. Arch. Otorhinolaryngol. 269: 1127-32.

Barkai G., D. Greenberg, N. Givon-Lavi, E. Dreifuss, D. Vardy and R. Dagan. 2005. Community prescribing and resistant Streptococcus pneumoniae. Emerg. Infect. Dis. 11: 829-837.

Bogaert D., R. de Groot and P.W.M. Hermans. 2004. Streptococcus pneumoniae colonization: the key to pneumococcal disease. The Lancet Infect. Dis. 4: 144-154.

Borer A., H. Meirson, N. Peled, N. Porat, R. Dagan, D. Fraser, J. Gilad, N. Zehavi and P. Yagupsky. 2001. Antibiotic-resistant pneumococci carried by young children do not appear to disseminate to adult members of a closed community. Clin. Infect. Dis. 33: 436-444. Brook I. and K. Shah. 2001. Bacteriology of adenoids and tonsils in children with recurrent adenotonsillitis. Ann. Otol. Rhinol. Laryngol. 110: 844-848.

Brook I., K. Shah and W. Jackson. 2000. Microbiology of healthy and diseased adenoids. Laryngoscope 110: 994-999.

Center K.J. 2007. Prevenar ${ }^{\text {ma }}$ vaccination: review of the global data, 2006. Vaccine 25: 3085-3089.

De Lencastre H. and A. Tomasz. 2002. From ecological reservoir to disease: the nasopharynx, day care centres and drug-resistant clones of Streptococcus pneumoniae. J. Antimicrob. Chemother. 50 (Suppl S2): 75-81.

Greenberg D., A. Broides, I. Blancovich, N. Peled, N. Givon-Lavi and R. Dagan. 2004. Relative importance of nasopharyngeal versus oropharyngeal sampling for isolation of Streptococcus pneumoniae and Haemophilus influenzae from healthy and sick individuals varies with age. J. Clin. Microbiol. 42: 4604-4609.

Greenberg D., N. Givon-Lavi, A. Broides, I. Blancovich, N. Peled and R. Dagan. 2006. The contribution of smoking and exposure to tobacco smoke to Streptococcus pneumoniae and Haemophilus influenzae carriage in children and their mothers. Clin. Infect. Dis. 42: 897-903.

Gryczynska D., J. Kobos and A. Zakrzewska. 1999. Relationship between passive smoking, recurrent respiratory tract infections and otitis media in children. Int. J. Pediatr. Otorhinolaryngol. 49 (suppl 1): S275-278.

Gunnarsson R.K., S.E. Holm and M. Soderstrom. 2001. The prevalence of potential pathogenic bacteria in nasopharyngeal samples from individuals with a respiratory tract infection and sore throat - implications for the diagnosis of pharyngotonsillitis. Family Practice. 18: 266-271.

Isaacman D.J., E.D. McIntosh and R.R. Reinert. 2010. Burden of invasive pneumococcal disease and serotype distribution among Streptococcus pneumoniae isolates in young children in Europe: impact of the 7-valent pneumococcal conjugate vaccine and considerations for the future conjugate vaccines. Int. J. Infect. Dis. 14: e197-e209.

Jacobs M.R., D. Felmingham, P.C. Appelbaum, R.N. Grüneberg and Alexander Project Group. 2003. The Alexander Project 1998-2000: susceptibility of pathogens isolated from communityacquired respiratory tract infection to commonly used antimicrobial agents. J. Antimicrob. Chemother. 52: 229-246.
Jeong J.H., D.W. Lee and R.A. Ryu. 2007. Bacteriologic comparison of tonsil core in recurrent tonsillitis and tonsillar hypertrophy. Laryngoscope 117: 2146-2151.

Karlidag T., K. Demirdag, I. Kaygusuz, M. Ozden, S. Yalcin and L. Ozturk. 2002. Resistant bacteria in the adenoid tissues of children with otitis media with effusion. Int. J. Pediatr. Otorhinolaryngol. 64: 35-40.

Kosikowska U., I. Korona-Glowniak and A. Malm. 2011. Passive smoking as a risk factor for upper respiratory tract colonization by Haemophilus influenzae in healthy pre-school children. Pol. J. Environ. Stud. 20: 1541-1545.

Lopalco P.L. 2009. Childhood pneumococcal vaccination in Europe. J. Prev. Med. Hyg. 50: 197-200.

McCay J.E. 2000. Resistant bacteria in the adenoids: a preliminary report. Arch. Otolaryngol. Head Neck Surg. 126: 625-629.

McIntosch E.D., B. Fritzell and M.A. Fletcher. 2007. Burden of paediatric invasive pneumococcal disease in Europe, 2005. Epidemiol. Infect.135: 644-56.

Niedzielski A., I. Korona-Glowniak and A. Malm. 2013. High prevalence of Streptococcus pneumoniae in adenoids and nasopharynx in preschool children with recurrent upper respiratory tract infections in Poland - distribution of serotypes and drug resistance patterns. Med. Sci. Monit.19: 54-60.

O'Brien K.L., L.J. Wolfson, J.P. Watt, E. Henkle, M. Deloria-Knoll, N. McCall, E. Lee, K. Mulholland, O.S. Levine, T. Cherian, for the Hib and Pneumococcal Global Burden of Disease Study Team. 2009. The global burden of disease due to Streptococcus pneumoniae in children less than 5 years of age. Lancet 374: 893-902.

Principi N., P. Marchisio, G.P. Schito and S. Mannelli. 1999. Risk factors for carriage of respiratory pathogens in the nasopharynx of healthy children. Pediatr. Infect. Dis. J. 18: 517-523.

Riedel S., S.E. Beekmann, K.P. Heilmann, S.S. Richter, J. Garciade-Lomas, M. Ferech, H. Goosens and G.V. Doern. 2007. Antimicrobial use in Europe and antimicrobial resistance in Streptococcus pneumoniae. Eur. J. Clin. Microbiol. Infect. Dis. 26: 485-490. Simoes A.S., C. Valente, H. de Lencastre and R. Sa-Leao. 2011. Rapid identification of noncapsulated Streptococcus pneumoniae in nasopharyngeal samples allowing detection of co-colonization and reevaluation of prevalence. Diagn. Microbiol. Infect. Dis. 71: 208-216.

Skoczyńska A., E. Sadowy, K. Bojarska, J. Strzelecki, A. Kuch, A. Gołębiewska, I. Waśko, M. Foryś, M. van der Linden and W. Hryniewicz. 2011. Participants of laboratory-based surveillance of community acquired invasive bacterial infections (BINet). The current status of invasive pneumococcal disease in Poland. Vaccine 29: 2199-205.

Syrjänen R.K., T.M. Kilpi, T.H. Kaijalainen, E.E. Herva and A.K. Takala. 2001. Nasopharyngeal carriage of Streptococcus pneumoniae in Finnish children younger than 2 years old. J. Infect. Dis. 184: 451-459.

van de Sande-Bruinsma N., H. Grundmann, D. Verloo, E. Tiemersma, J. Monen, H. Goossens, M. Ferech, the European Antimicrobial Resistance Surveillance System and European Surveillance of Antimicrobial Consumption Project Groups. 2008. Antimicrobial drug use and resistance in Europe. Emerg. Infect. Dis. 14: $1722-1730$

World Health Organization. 2007. Pneumococcal conjugate vaccine for childhood immunization - WHO position paper. Wkly Epidemiol. Rec. 12: 93-104. 